\title{
EFFECT OF SUGARED CHEWING GUM VERSUS SUGAR FREE CHEWING GUM AFTER ACIDIC CHALLENGE ON CARIES PROFILE OF YOUNG ADULTS USING CARIOGRAM. A RANDOMIZED CONTROL TRIAL
}

\author{
Rania Sayed Mosallama* and Mona Shabaan **
}

\begin{abstract}
Objective: The present study was conducted to assess the effect of sugar containing and sugar free chewing gums, after candy intake, on caries profile of young adults using Cariogram software.

Methods: Thirty dental students, 20-25 years old, were randomly selected and were divided into two groups according to type of chewing gum used either sugared gum or sugar free gum. Related general diseases, Diet contents, Diet frequency, Fluoride program, Clinical judgment and Saliva chairside tests (to record the flow rate and buffer capacity) were performed at baseline, after candy intake and after chewing gum. Based on these data, caries-related variables were recorded into the Cariogram software to calculate the predicted chance of avoiding caries for each subject.
\end{abstract}

Results: In each group, there was a statistically significant difference between baseline, after candy and after chewing gum regarding salivary secretion, buffering capacity and actual chance. Comparing between two groups, there was a statistically significant difference between two groups regarding salivary secretion while there was no statistically significance difference regarding buffering capacity and actual chance.

Conclusion: Chewing gum can be used as short-term preventive strategies to neutralize salivary $\mathrm{pH}$ after candy intake thus reducing caries risk in young adults.

KEYWORDS: Candy, Sugared gum, Sugar free gum, Salivary pH, Salivary secretion rate, Actual chance, Cariogram.

\section{INTRODUCTION}

Dental caries is the most prevalent disease that affect $60-90 \%$ of schoolchildren and wide majority of adults ${ }^{(1)}$. Individuals are susceptible to dental caries throughout their lifetime. There are four main contributing factors for dental caries: diet, microflora, a susceptible tooth surface and time ${ }^{(2)}$. Diet is a dominant factor of dental caries that can

* Ass.Prof. Department of Conservative Dentistry, Faculty of Dentistry, Cairo University.

** Lecturer Department of Operative Dentistry, Faculty of Oral and Dental Medicine, Ahram Canadian University. 
mask other factors ${ }^{(3)}$. Modern life style requires short break times and fast meals. The use of junk food and snaking between meals is commonly seen in the younger age group ${ }^{(1,4)}$. Candy and similar products became dessert or snack food. Candy is basically sugar dissolved in water and concentrated through boiling. With more heat, more of the water evaporates and the result is generally the hard and brittle types of candies, while soft candies are made in lower temperatures. Most people like to eat candy now and then but doing so regularly as negative effects on oral health ${ }^{(5,6)}$. This highlight the importance of caries preventive strategies that requires less time and efforts to improve public oral health conditions.

Preventive strategies are considered as top priority in oral health programs. Based on available evidence, interventions that require people to spend minimum time and make minor effort dramatically have improved public oral health condition. On the contrary, interventions which need active and longtime participation of people were shown to be less successful in making a steady decline in caries incidence $^{(7)}$.

Chewing gum is one of the short-term caries preventive strategies. Chewing gum is a unique food because it is chewed for a long time with a few calories. Gum are manufactured in many different shapes, sizes and flavors. It can be used as an adjunctive method of mechanical control of bacterial plaque in addition to tooth brushing. Moreover, it has many beneficial effects such as salivary stimulation, promote remineralization and $\mathrm{pH}$ regulation ${ }^{(8)}$. It has a practical recommendation for both children and adults. So, most of oral hygienist advised their patients for chewing gum immediately after meals especially for those with high caries index. In the last decade, sugar-free chewing gum has been claimed to provide oral health benefits, including caries reduction and plaque control (9-13). It is produced by replacing sucrose by sugar alcohol such as glycitols, polyalcohol or polyols ${ }^{(14,15)}$.
Different caries risk assessment and evaluation models have been developed in recent years. The risk profile is an important factor in decision-making processes for caries prevention and management ${ }^{(16)}$. Cariogram is an interactive computerized program that illustrate the multifactorial aspects of dental caries graphically. It also illustrates to what extent various factors affect the chance to avoid new caries. In addition, this educational interactive program has been developed to act as a guide to assess an individual's caries profile and to encourage preventive measures to be introduced before new cavities could develop. ${ }^{(17-21)}$.

Lack of any supporting evidence for such claim led us to carry out the present study, which compares the efficacy of gum chewing in improving caries profile after candy intake. This study will benefit preventive dentistry as the main goal is to prevent rather than cure. The objectives of this study were to assess effect of short-term preventive measures on the caries risk profile in dentistry students after candy intake expressed by the likelihood of avoiding new caries using Cariogram. So, the null hypothesis tested is that sugared chewing gum and sugar-free chewing gum will have no effect on caries profile using Cariogram.

\section{MATERIAL AND METHODS}

\section{Ethical Considerations}

The study approval was given by ethics committee of Cairo University, Faculty of Oral and Dental Medicine, Egypt (registration number 189-73). Before carrying out the study, the purpose and methodology of the study were clarified to each participant and informed consent form was obtained.

\section{Sample selection}

A parallel randomized control trial was done on total of 30 dental students from Ahram Canadian University, Faculty of Oral and Dental Medicine, 
who volunteered to participate in this study. The inclusion criteria were that subjects should be medically healthy, had full permeant dentition, aged 20-25 years. The participants with the presence of active dental or periodontal diseases, prosthesis, TMJ disorders and wearing orthodontic appliances were excluded from the study.

\section{Study design}

Subjects were randomly allocated into two groups comprising of 15 subject each: group A: chewing sugared gum group, group B: chewing sugar free gum group. The chewing gums used for the study were Trident (sugar-free gum and Chiclets (sugar containing gum). They were purchased from general stores. All data regarding the tested materials were tabulated in (Table 1).

\section{Saliva sampling}

According to the experimental protocol, the participants were informed not to eat at least 1 hour before starting the procedures. Samples were collected in the morning between 10-11am under standardized conditions. The stimulated salivary sample was collected while chewing on a piece of paraffin wax for 5 minutes in a test tube gradu- ated in millimeters and the salivary secretion rate was estimated as milliliters per minutes $(\mathrm{ml} / \mathrm{min})$. The buffer capacity was measured immediately using the digital $\mathrm{pH}$ meter (Pen type $\mathrm{pH}$ meter,pH-009(1), Pometer China). The accuracy of $\mathrm{pH}$ meter was checked at regular intervals to ensure that readings were accurate. Again, the stimulated salivary sample was collected after chewing candy for 2 minutes and the buffer capacity and the salivary secretion rate were evaluated. After that 15 subjects were asked to chew a sugar containing gum and the others chewed sugar free gum for 3 minutes with no swallowing and the sample was collected and the buffer capacity salivary secretion rate were measured again. Recording of the data was done by a welltrained recorder who recorded data on a pro form containing details of the selected test material of each study subject. To minimize bias in the data, an independent observer, blinded to the study's aim, recorded all the .readings

\section{Creating risk profile of Cariogram:}

A caries risk profile of each individual was

TABLE (1): Materials specifications, chemical composition and manufacturer.

\begin{tabular}{|l|l|l|}
\hline Material & Ingredients & Manufacturer \\
\hline Candy (Toffiy) & $\begin{array}{l}\text { sugar, glucose hydrogenated vegetable oil, edible beef gelatin, fruit juice } \\
\text { concentrates (apple, orange, strawberry, cherry, watermelon), pectin, acid } \\
\text { regulator citric acid, flavors (apple, strawberry, cherry, orange, watermelon), } \\
\text { emulsifier soya lecithin. Contains milk, egg, hazelnut }\end{array}$ & Prof Turkey \\
\hline $\begin{array}{l}\text { Sugar } \\
\text { chewing gum (Chiclets) }\end{array}$ & $\begin{array}{l}\text { Nugar, Gum Base, Glucose Syrup, Modified Food Starch, Artificial and } \\
\text { Natavoring, Corn Oil, Candelilla Wax, BHT (to Maintain Freshness), }\end{array}$ & Cadbury Adams \\
\hline $\begin{array}{l}\text { Sugar-free chewing gum } \\
\text { (Trident) }\end{array}$ & $\begin{array}{l}\text { Sorbitol, Gum Base, Xylitol, Glycerin, Natural and Artificial Flavoring, } \\
\text { Mannitol, less than 2\% of: Acesulfame Potassium, Aspartame, BHT (to } \\
\text { Maintain Freshness), Red 40 Lake and Soy Lecithin }\end{array}$ & $\begin{array}{l}\text { United Kingdom } \\
\text { the }\end{array}$ \\
\hline
\end{tabular}


obtained at each of three tested steps using Cariogram software. For each subject, six caries related variables were put into the Cariogram and the whole collected data were scored according to the predetermined scale as $0,1,2$ or 3 . For all individuals, the "clinical judgment" factor was given a sore of one. The scores were entered into the Cariogram computer software program to calculate the 'caries risk' and so the 'chance of avoid-

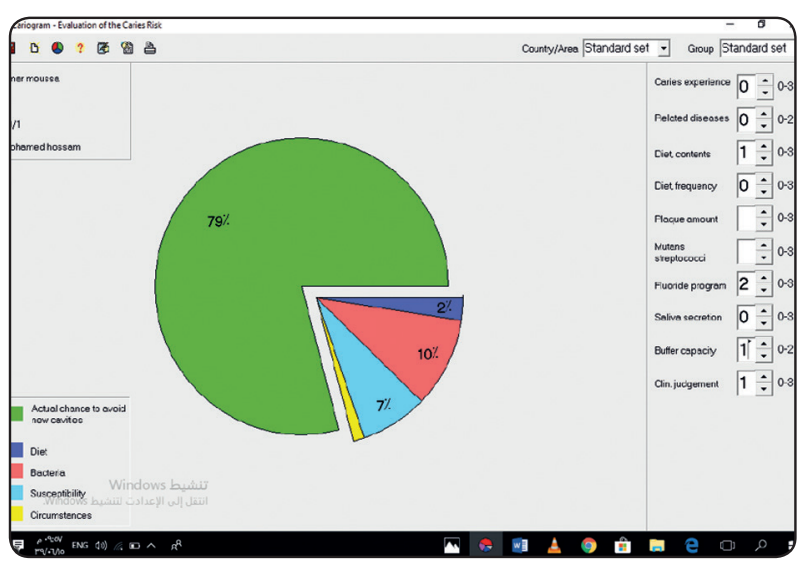

Fig. (1) Example of Cariogram from the present study showing $79 \%$ chance of avoiding new caries

ance of new caries' for each subject Finally, the caries profile for each subject was obtained as a pie chart with five colored sectors, which revealed the chance of avoiding caries as a percentage (Figure 1)

\section{Statistical analysis}

The mean and standard deviation values were calculated for each group in each test. Data were explored for normality using Kolmogorov-Smirnov and Shapiro-Wilk tests and showed parametric (normal) distribution. One-way ANOVA followed by Tukey post hoc test was used to compare between groups. The significance level was set at $\mathrm{P} \leq 0.05$.
Statistical analysis was performed with IBM® SPSS® Statistics Version 20 for Windows.

\section{RESULTS}

For sugared gum group, there was a statistically significant difference between baseline, after candy and after sugar gum intake for salivary secretion, buffering and actual chance $(p<0.001)$ (Table 2 , Figure 2). The highest mean value for secretion was found in after candy followed after sugared gum while the least mean value was found in baseline. The highest mean value for buffer capacity was found in baseline followed by sugared gum while the least mean value was found after candy. The highest actual chance was after sugared gum, while no statistically significant difference was found between baseline and after candy where $(p=0.447)$.

Regarding sugar free gum, there was a statistically significant difference between baseline, after candy and after treatment for salivary secretion, buffer capacity and actual chance where $(p<0.001)$ (Table 3 , Figure 3). The highest mean value for secretion was found in after candy followed by after sugar free gum while the least mean value was found in baseline. However, the highest mean value for buffering was found in baseline followed by after sugar free gum while the least mean value was found in after candy. In addition, the highest mean value for actual chance was found in after sugar free chewing gum and no statistically significant difference was found between baseline and after candy where $(p=0.760)$.

Comparing all the tested materials, there was a statistically significant difference between two groups regarding salivary secretion only $(p<0.001)$ 
TABLE (2): The mean, standard deviation (SD) values of sugared gum group.

\begin{tabular}{|c|c|c|c|c|c|c|}
\hline \multirow[t]{3}{*}{ Variables } & \multicolumn{6}{|l|}{ Sugar gum } \\
\hline & \multicolumn{2}{|l|}{ Saliva secretion } & \multicolumn{2}{|c|}{ Saliva buffer capacity } & \multicolumn{2}{|l|}{ Actual chance } \\
\hline & Mean & SD & Mean & SD & Mean & SD \\
\hline Baseline & $1.09^{\mathrm{c}}$ & 0.38 & $7.97^{\mathrm{a}}$ & 0.36 & $51.40^{\mathrm{b}}$ & 10.61 \\
\hline After candy & $4.19^{\mathrm{a}}$ & 0.90 & $6.13^{c}$ & 0.58 & $52.67^{\mathrm{b}}$ & 5.85 \\
\hline After chewing & $2.40^{\mathrm{b}}$ & 0.45 & $7.16^{b}$ & 0.47 & $55.27^{\mathrm{a}}$ & 7.20 \\
\hline p-value & & $<0.001 *$ & & $<0.001 *$ & & $0.032 *$ \\
\hline
\end{tabular}

Means with different small letters in the same column indicate statistically significance difference. *; significant $(p<0.05)$ ns; non-significant ( $p>0.05)$

Table (3): The mean, standard deviation (SD) values of sugar free gum group.

\begin{tabular}{|c|c|c|c|c|c|c|}
\hline \multirow[t]{3}{*}{ Variables } & \multicolumn{6}{|r|}{ Sugar free gum } \\
\hline & \multicolumn{2}{|r|}{ Saliva secretion } & \multicolumn{2}{|c|}{ Saliva buffer capacity } & \multicolumn{2}{|r|}{ Actual chance } \\
\hline & Mean & SD & Mean & SD & Mean & SD \\
\hline Baseline & $1.33^{\mathrm{c}}$ & 0.43 & $7.55^{\mathrm{a}}$ & 0.81 & $47.73^{b}$ & 10.65 \\
\hline After candy & $4.81^{\mathrm{a}}$ & 0.89 & $5.91^{\mathrm{c}}$ & 0.53 & $48.20^{\mathrm{b}}$ & 11.92 \\
\hline After chewing & $2.97^{b}$ & 0.73 & $6.97^{b}$ & 0.49 & $51.47^{\mathrm{a}}$ & 11.11 \\
\hline p-value & \multicolumn{2}{|c|}{$<0.001 *$} & $<0.001 *$ & & \multicolumn{2}{|l|}{$0.016 *$} \\
\hline
\end{tabular}

Means with different small letters in the same column indicate statistically significance difference. *; significant $(\mathrm{p}<0.05) \quad \mathrm{ns} ;$ non-significant $(\mathrm{p}>0.05)$

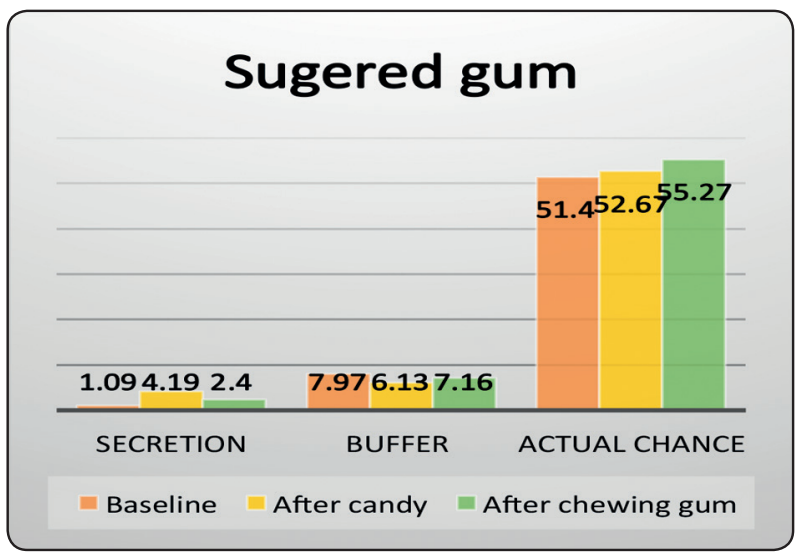

Fig. (2): Bar chart representing salivary secretion, buffer capacity and actual chance of sugared gum group.

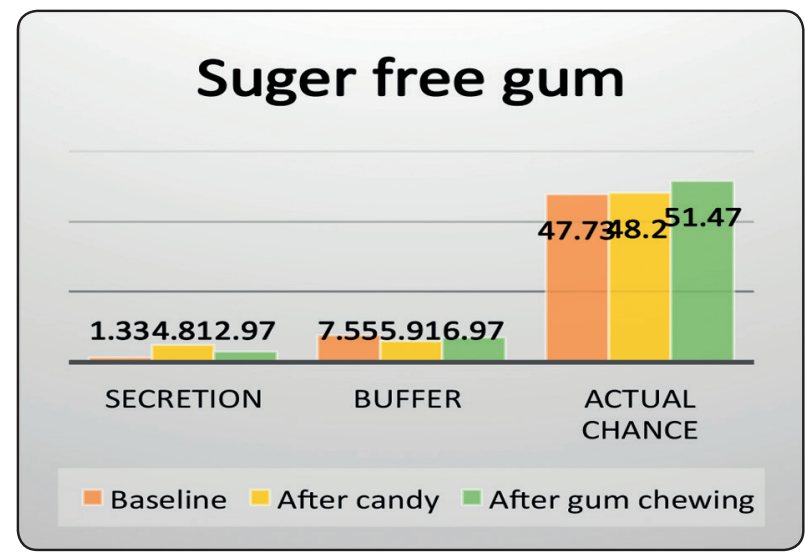

Fig. (3): Bar chart representing salivary secretion, buffer capacity and actual chance of sugar free gum group. 
(Table 4). The highest mean for salivary secretion value was found in sugar free gum followed by sugared gum. Regarding buffering capacity and actual chance, there was no statistically significant difference found between all groups $(p>0.05)$. The highest mean value was found in sugared gum while the least mean value was found in sugar free gum.

\section{DISCUSSION}

Modern lifestyle mandates short break times and quick meals with less frequency of oral hygiene practice. Physical status of food plays a very important role in its cariogenic potential. Sticky and solid sugars get stuck to the teeth surface compared to liquid sugars which pass through the oral cavity with limited contact time ${ }^{(22)}$. Hence, this study was undertaken with the aim of assessing the changes in salivary $\mathrm{pH}$, flow and actual chance to avoid new caries after candy intake and to evaluate different oral hygiene practice that takes less time and efforts.

Chewing gum is a habit practiced regularly by a relatively high portion of individuals. The ability of chewing gum to aid in caries control comes from the chewing action itself which stimulates saliva flow, increases the buffering capacity and thus neutralizes the acidic $\mathrm{pH}^{(12,23,24)}$. For this reason, we evaluated a free sugar and sugar containing chewing gums to observe the difference between them on the salivary secretion rate, salivary $\mathrm{pH}$ and the actual chance to avoid caries.

The Cariogram has been used extensively in several studies and has demonstrated fairly high efficacy and good reliability as reported by many authors. It is an objective, quantitative method that uses a software computer program to calculate the data ${ }^{(17,20,21)}$. The main purpose of the using Cariogram in this study is to demonstrate the caries risk expressed as the chance to avoid new caries. In addition, it can be used effectively to improve the comprehension of the factors that are having or could have a negative effect through the pie chart presentation with different sectors. For salivary $\mathrm{pH}$ evaluation, a digital $\mathrm{pH}$ meter was used which is a quick and convenient method to measure $\mathrm{PH}$ levels of liquids. In addition, this PH meter is reliable, portable, sensitive and able to give a quick response of $\mathrm{PH}$.

The results of the present study showed that the use of different chewing gums decreases the subject's caries risk after candy intake. A statistically significant change in the caries risk as evaluated by the Cariogram software was observed regarding chance of avoiding caries in comparison to candy intake. Candy intake, results revealed that there was a significant increase in salivary secretion and decrease in salivary $\mathrm{pH}$ after candy intake. This could be attributed to its extended contact time and adherence to tooth surface. Also, candy is considered as a slowly dissolving sugar that have extended exposure time in the oral cavity as result of gradual release of sugars during consumption ${ }^{(22)}$. Hans et al. ${ }^{(22)}$ reported that carbohydrates consumed in liquid form usually do not stay in mouth very long while if teeth are constantly exposed to sugary drinks, the acids produced by bacteria remains for aa longer time in the oral cavity.

This study shows a statistically significant increase in salivary secretion with both chewing gum. Chewing gums have an anti-decay effect through various mechanisms. It enhances tooth cleansing by stimulating saliva secretions through chewing process and chemical stimulation of taste buds ${ }^{(23)}$. In addition, chewing gums cause temporary rise in salivary secretion which affect the buffering capacity thereby causing neutralization and rise in pH. ${ }^{(24)}$. The data showed that both chewing gums elevated saliva $\mathrm{pH}$ beyond the resting $\mathrm{pH}$ value. Lif Hologerson, et al. ${ }^{(25)}$ and Akay, et al. ${ }^{(26)}$, examined the effects of various gums on dental plaque $\mathrm{pH}$ came to the conclusion that chewing gum increases dental plaque $\mathrm{pH}$. However, sugared gum showed a significant increase in salivary secretion compared 
to sugar free gum. Cochrance et al. ${ }^{(27)}$ reported that chewing gums have meaningful reminerlizing effect on enamel with sugar free gum containing casein phosphopeptide amorphous calcium phosphate. Chewing sugar-free gums arise the salivary $\mathrm{PH}$ because of inclusion of polyols like xylitol into gums that improve the oral health benefits. Moreover, chewing gums containing xylitol as sweeteners have anti-bacterial effect. ${ }^{(28,29)}$ The study conducted by Tangade et al (11) reported minimal difference between the sugar free and sugar containing gum. Ingle et al. ${ }^{(12)}$ reported sugar free group had a lesser mean plaque score as compared to sugar containing group. The difference in results may be attributed to using of different snakes, different times of gum chewing and different ingredients.

This study suggests that, the use of short-term preventive measures such as gum chewing as an adjunct to mechanical measures can be considered as practical recommendation for caries prevention. However, further studies need to be carried out to evaluate the influence of chewing practice on the composition of saliva. Also, further studies are recommended to be done on larger samples with wider geographical representations.

\section{CONCLUSION}

Chewing gum can be used as short-term preventive strategies to neutralize salivary $\mathrm{pH}$ after candy intake thus reducing caries risk in young adult.

\section{ACKNOWLEDGMENT}

The authors would like to thank Dr.Nawal Eidaros and Dr. Doaa Gamal, Ass. Lecturer in Conservative Dentistry Department, Ahram Canadian University, for assisting in study design, Rana Hosam Eldin, Nermeen Aslan (Fifth year student, Ahram Canadian University, Giza, Egypt) for participation in the clinical work for partial fulfillment of the graduation requirements. The author would like also to thank all participants for their cooperation.

\section{REFERENCES}

1. Demircia M, Tuncera S, Yuceokurb A. Prevalence of caries on individual tooth surfaces and its distribution by age and gender in university clinic patients. European Journal of Dentistry. 2010;4:270-279.

2. Newborn E. Cariology 3rd ed, p.29 Quintessenz 1989.

3. Moynihan P. The interrelationship between diet and oral health. Proceedings of the Nutrition Society. 2005; 64(4):571-580.

4. Nielsen SJ, Popkin BM. Changes in beverage intake between 1977 and 2001. American Journal of Preventive Medicine. 2004;27(3):205-210.

5. Gupta P, Gupta N, Pawar A, Biraidar S, Natt A, Singh H. Role of Sugar and Sugar Substitutes in Dental Caries: A Review. ISRN Dentistry.2013; 1-5.

6. Azrak B, Willershausen B, Meyer N, Callaway A. Course of changes in salivary $\mathrm{pH}$-values after intake of different beverages in young children. Oral Health Prev Dent. 2008;6(2):159-64.

7. Watt R. Strategies and approaches in oral disease prevention and health promotion.Bull.World Health Organ. 2005; 83:711-718.

8. Imfeld T. Chewing Gum-Facts and Fiction: A Review of Gum-Chewing and Oral Health. Crit Rev Oral Biol Med 1999; 10:405-19.

9. Szoke J, Banoczy J, Proskin HM. Effect of after-meal sucrose-free gum-chewing on clinical caries. J Dent Res. 2001; 80: 1725-1729.

10. Liop M, Jimeno G, Acien M, Dlmau B. Effect of xylitol chewing gum on salivary rate, $\mathrm{pH}$, buffering capacity and presence of Streptococcus mutance in saliva. European Journal of Pediatric Dentistry. 2010;11: 9-14.

11. Tangade P, Mathur A, Chaudhary S, Gupta R. The Effect of Sugar Free and Sugar Chewing Gums on Plaque Deposition. Dental Research Journal. 2012; 9:309-13.

12. Ingle $\mathrm{N}$, Dubey $\mathrm{H}$, Navpreet $\mathrm{KN}$, Gupta R. Effect of sugared and sugar free chewing gums on dental plaque: $\mathrm{A}$ clinical study. Journal of Advanced Oral Research. 2013; 4: 8-10.

13. Mirzakhani M, Siavashani M, Akhavan P. The Effect of Different Chewing Gum on pH of Dental Plaque. Journal of Dental School. 2014; 32(1): 45-52 .

14. Brian BA. The use of sorbitol and xylitol sweetened chewing gum in caries control. JADA 2006;137:190-96.

15. Kauko MK. Sugar Alcohols, Caries Incidence, and Remineralization of Caries Lesions: A Literature Review. International Journal of Dentistry 2010;8:1-23. 
16. Bratthall D, Petersson GH, Stjernsward JR. Assessment of Caries Risk in the Clinic-a modern Approach. In Advances in Operative Dentistry. Ed: Wilson NHF, Roulet JF, Fuzzi M. Quintessence Publishing Co, Inc. 2001; 2: 61-72.

17. Petersson GH, Twetman S, Bratthall D. Evaluation of a computer program for caries risk assessment in schoolchildren. Caries Research. 2002;36;327-340.

18. Petersson GH, Bratthall D. Caries risk assessment: a comparison between the computer program "Cariogram", dental hygienists and dentists. Swe Dent J 2000; 24:12937.

19. Petersson GH. Assessing caries risk using the Cariogram model. Swe Dent J Supplement 2003; 158:1

20. Bratthall D, Petersson GH. Cariogram-a multifactorial risk assessment model for a multifactorial disease. Community Dent Oral Epidemiology. 2005;33:256-64.

21. Utreja D, Simratvir M, Kaur A, Kwatra KS, Singh P, Dua V. An evaluation of the Cariogram as a predictor model. Int Dent J. 2010;60:282-4.

22. Hans R, Thomas S, Garla B, Dagli R, Hans M. Effect of Various Sugary Beverages on Salivary pH, Flow Rate, and Oral Clearance Rate amongst Adults. Hindawi Publishing Corporation Scientifica. 2016 : 1-6.

23. Dodds MW. The oral health benefits of chewing gum. J. Iran Dent. Assoc. 2013; 58(5):253-261.
24. Polland KE, Higgins F, Orchardson R. Salivary flow rate and $\mathrm{pH}$ during prolonged gum chewing in humans. J. Oral Rehabil. 2003; 30(9):861-865.

25. Hologerson PL, Stecksen-Blicks C, Sjostrom I, Twetman S. Effect of Xylitol-containing chewing gums on interdental plaque- $\mathrm{pH}$ in habitual Xylitol consumers. Acta Odontol Scand. 2005; 63: 233-238.

26. Akay G, Altay N, Galis U. Effect of chewing gum containing calcium-Flouride on salivary acidogenicity. Caries Res 2007; 41: 208-211.

27. Cochrane NJ, Shen P, Byrne SJ, Walker GD, Adams GG, Yuan Y, Reynolds C, Hoffmann B, Dashper SG, Reynolds EC. Remineralization by chewing sugar-free gums in a randomized, controlled in situ trial including dietary intake and gauze to promote plaque formation. Caries Res. 2012; 46(2):147-155.

28. Llop MR, Jimeno FG, Acien RM, Dalmau LJ . Effects of xylitol chewing gum on salivary flow rate, $\mathrm{pH}$, buffering capacity and presence of Streptococcus mutans in saliva. Eur. J. Pediatric. Dent. 2010; 11(1):9-14.

29. Hanno AG, Alamoudi NM, Almushayt AS, Masoud MI, Sabbagh HJ, Farsi NM . Effect of xylitol on dental caries and salivary Streptococcus mutans levels among a group of mother-child pairs. J. Clin. Pediatr. Dent. 2012; 36(1):25-30. 